\title{
Direct Ambient Analysis of Pharmaceutical and Ecstasy Tablets
}

\author{
Luc Alexis Leuthold§a , Jean-François Mandscheffa ${ }^{a}$ Marc Fathi ${ }^{b}$, Christian Giroud ${ }^{c}$, Marc Augsburger ${ }^{c}$, \\ Emmanuel Varesio ${ }^{a}$, and Gérard Hopfgartner*a \\ §METTLER TOLEDO Award Winner (Oral Presentation)
}

\begin{abstract}
Desorption electrospray ionization (DESI) is a new ionization technique recently introduced in analytical chemistry for the ambient analysis of surfaces by mass spectrometry. We present here new developments in this field, focusing on their use for the analysis of solid forms, as pharmaceutical tablets or illicit drug tablets. Results recently published by ourselves are discussed in the context of related publications.
\end{abstract}

Keywords: Ambient analysis · Desorption electrospray ionization · Mass spectrometry · Pharmaceutical analysis - Toxicological screening

\section{Introduction}

For the analytical chemist, direct analysis of samples is the ultimate goal, which is however sometimes difficult to achieve. Direct analysis means the direct introduction of the sample in the analytical instrument or platform, without any sample preparation. Despite the current instrumentation available and major advances in analytical chemistry in the last decade, direct analysis remains a challenging task. The balance is often be-

${ }^{\star}$ Correspondence: Prof. G. Hopfgartnera

Tel.: +412237963 44

Fax: +412237968 08

E-Mail: gerard.hopfgartner@pharm.unige.ch

aLife Sciences Mass Spectrometry

School of Pharmaceutical Sciences

EPGL, University of Geneva

20, Bd d'Yvoy

$\mathrm{CH}-1211$ Genève 4

${ }^{\mathrm{b}}$ Geneva University Hospital

Clinical Chemistry Central Laboratory

Toxicology Section

Micheli-Du-Crest 24

$\mathrm{CH}-1211$ Genève 14

cForensic Chemistry and Toxicology Laboratory

Institute of Legal Medicine

University of Lausanne

Rue du Bugnon 21

$\mathrm{CH}-1005$ Lausanne tween information obtained and analysis time or complexity: where rapid analysis is a priority, the quality of information often suffers. Either the usual sample preparation and separation times, prior to detection, are reduced to the minimum, or the sample is analyzed directly. Both approaches present challenges in terms of the specificity (the analyte can be present among others) and sensitivity (the matrix can decrease the intensity for the response of the analyte, a phenomenon known as 'matrix suppression') needed for the detection.

From the wide range of detection techniques, mass spectrometry (MS) has gained much attention since its introduction and is now becoming one of the most widely used detection techniques in pharmaceutical, toxicological or environmental analysis. With the introduction of desorption techniques, the most common being matrixassisted laser desorption (MALDI), it has become possible to sample surfaces by MS. Previously, desorption ionization (DI) techniques were performed in a high-vacuum environment, but only six years ago DI experiments were carried out in ambient environment; atmospheric pressure MALDI being an ionization technique in which a solid sample could be examined at atmospheric pressure [1][2]. Recording mass spectra of ordinary samples in the laboratory or in the field, while simultaneous chemical or physical modifications could be performed during their analysis, could be one of the next major qualitative advances in MS.

Desorption electrospray ionization (DESI) is an atmospheric pressure desorption ionization method introduced by Cooks and coworkers that produces ions directly from the surface to be analyzed. The ions then are sampled with the mass spectrometer [3]. DESI is based on charged liquid droplets that are directed, with a high velocity gas jet $(\mathrm{ca} .300 \mathrm{~m} / \mathrm{s})$, onto the surface to be analyzed. Analytes are desorbed from the surface and analyzed by the mass spectrometer. Compared to pressure MALDI, no matrix is needed to perform the experiment; a common advantage with laser desorption from porous silicon [4].

Other acronyms have been introduced in the meanwhile, describing related ionization techniques. Direct analysis in real time (DART) has been reported by Cody et al. [5]. This technique is based on the reactions of electronic or vibronic excitedstate species (metastable helium or nitrogen molecules) with reagent molecules and analytes. Both DESI and DART techniques allow surface analysis, DESI with a stream of electrosprayed liquid solvent and DART with a stream of excited gas. Using thermal vaporization by a hot nitrogen gas stream flowing from a probe, atmospheric-pressure solids analysis probe (ASAP) provides a way to analyze volatile and semi-volatile compounds [6]. The thermally induced vapors are ionized by corona discharge under standard APCI conditions. Its ability to obtain ions from biological tissue, currency and other objects placed in the path of the hot nitrogen stream was demonstrated. Commercial ionization sources were used after only slight modification, i.e. installation of a port for inserting the sample into the hot gas stream within the ion source region. Even more recently, a variant of 
DESI, electrospray-assisted laser desorption/ionization (ELDI), was described [7]. This combines laser desorption with postionization by electrospray for the rapid analysis of solid materials under ambient conditions. With this system, desorption and ionization can be independently controlled, which is not the case in DESI.

In the context of toxicological qualitative analysis, the analysis of drug or illicit tablets is one simple application where direct analysis would be useful. Since the sample to be analyzed is expected to be quite simple regarding the number of main components - no profiling of impurities has to be done - the question could be "are separation steps like gas or liquid chromatography, or even sample preparation, really mandatory?" In emergency departments of hospitals where patients can arrive with unknown tablets in their pockets, an almost instant analysis of the tablets, but with very high specificity, could save precious time for diagnostics. The rapid screening of illicit tablets could also find uses, since speed of analysis is not driven by emergency but by throughput, enabling more analysis in less time. Visual evaluation of the tablet (color, thickness, diameter, logo, cleavage, smell), comparing for example, Ecstasy tablets with a table of pictures from various sources, can help, but is not reliable at all. Small homemade series can involve frequent changes in appearance [8]. With abuse of commercial drugs, rapid identification is hindered by similarity in appearance of tablets and the identifier (name, brand code or number) not always found on the tablet.

The current way to analyze commercial drugs or illicit tablets is to dissolve them in an appropriate solvent, to filtrate or centrifuge the solution and to perform a separation with liquid chromatography (LC) coupled to a UV diode array detector (UV-DAD) or with gas chromatography (GC) coupled to a mass spectrometer. In the case of GC, sample preparation with derivatization is sometimes required. Some automated drug profiling systems were routinely used in emergency toxicology, such as the LC-UV REMEDi HS system [9]. If greater selectivity is needed, MS is generally used. UV-DAD or MS spectra are then compared to libraries of known compounds to identify the compounds in the tablet. The sample preparation and separation step required with these methods are time-consuming and it requires equipment for chromatography which can be a drawback in terms of ease of use. Flow injection analysis (FIA) or infusion of the liquid sample by a syringe pump into the source of the MS are alternatives that do not require chromatographic equipment, but still require solubilization of the sample, which is an additional preparation step when starting from solid forms.

For toxicological screening, some surface analysis techniques have been developed to identify or quantify analytes in unknown tablets. Infrared (IR) [10], near infrared (NIR) [11][12], Raman [13] and fluorescence [14][15] spectroscopy have been described for the analysis of pharmaceutical solid forms or illicit drugs, such as Ecstasy tablets. Other techniques like nuclear quadrupole resonance (NQR) also show perspectives as non-destructive analysis techniques [16]. The main advantage of these methods is still that no sample preparation and chromatographic separation are performed, thus dramatically shortening the analysis time, but specificities are lower compared to MS.
In this context, the question was to see how DESI-MS could complement the analytical tool collection currently available and we chose the application of formulated solids as a starting point for this investigation. We recently published results of DESI-MS used for the rapid and direct ambient analysis of a large variety of compounds relevant for toxicological screening purposes [17]. The home-made source and experimental set-up were described as well as a method of analyzing commercial drugs, illicit Ecstasy tablets, and compressed powders. Here we discuss these results in relation to other publications in the same field at this early stage of development.

\section{DESI-MS for the Analysis of Pharmaceutical Tablets}

Up to now, there are around a dozen publications on DESI-MS. After the initial publication [3], recent papers reported its use for the coupling of thin-layer chromatography and mass spectrometry [18], the detection of explosives [19][20], the mass spectrometric profiling of intact biological tissue [21], the characterization of the active ingredients in pharmaceutical samples formulated as tablets [17][22-24], ointments [22][24], gels [24] and liquids [22], the rapid in situ detection of alkaloids in plant tissues [25], the rapid analysis of Ecstasy tablets [17][26], the sampling of cannabis plant material [26], and the analysis of some pharmaceuticals and their glucuronide metabolites [27]. Instrumentation, mechanisms and applications in forensics, chemistry, and biology have been reviewed recently [28].

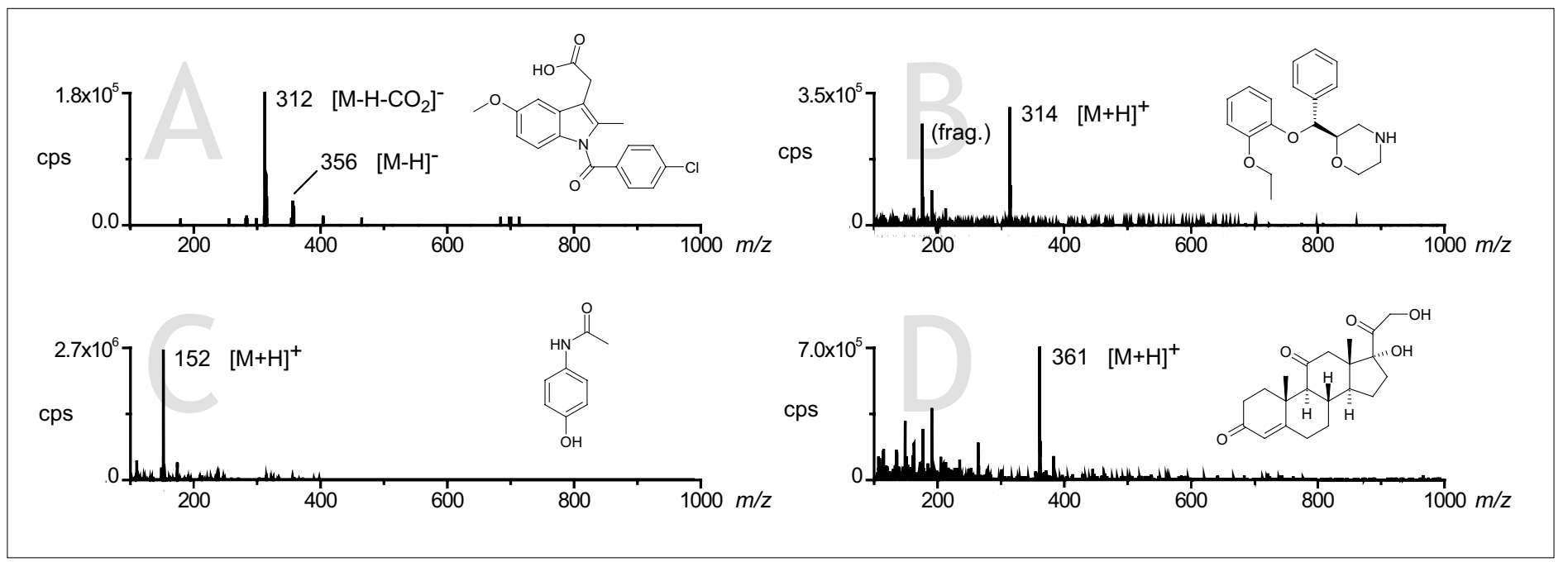

Fig.1. Typical DESI-EMS spectra of drug tablets, without background subtraction. (A) Indocid ${ }^{\circledR}$ tablet, containing $11 \%(\mathrm{w} / \mathrm{w})$ of indometacin $(\mathrm{MW}=357$ $\mathrm{Da}$ ), in negative mode. (B) Edronax ${ }^{\circledR}$ tablet, containing $1.9 \%(\mathrm{w} / \mathrm{w})$ reboxetin (MW $\left.=313 \mathrm{Da}\right)$, in positive mode. (C) Acetalgin ${ }^{\circledR}$ tablet, containing $67 \%(\mathrm{w} / \mathrm{w})$ paracetamol ( $\mathrm{MW}=151 \mathrm{Da})$, in positive mode. (D) Home-made tablet, containing $20 \%(\mathrm{w} / \mathrm{w})$ cortisone $(\mathrm{MW}=360 \mathrm{Da})$ with some cellulose as excipient, in positive mode. 


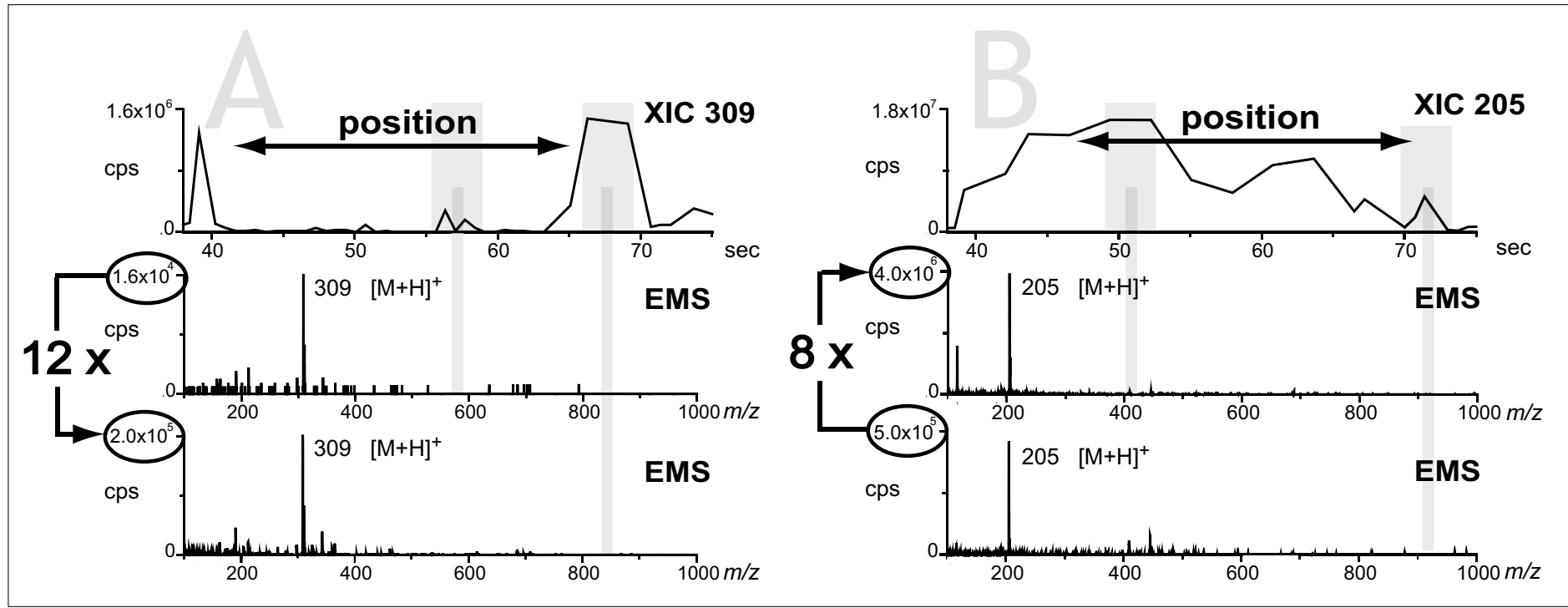

Fig. 2. DESI-MS in function of the position of the tablet under the spray. (A) Xanax ${ }^{\circledR}$ tablet, containing $0.8 \%(\mathrm{w} / \mathrm{w})$ of alprazolam (MW $\left.=308 \mathrm{Da}\right)$, analyzed in positive mode. (B) Servambutol ${ }^{\circledR}$ tablet, containing $71 \%(\mathrm{w} / \mathrm{w})$ of ethambutol $(\mathrm{MW}=204 \mathrm{Da})$, analyzed in positive mode. No background subtraction.

Amongst these first applications, the analysis of solid forms as pharmaceutical tablets has attracted much interest. DESI sources have been coupled to either a triple quadrupole tandem mass spectrometer [17], a time-of-flight [24] or an ion-mobility spectrometer coupled to a quadrupole-timeof-flight mass spectrometer [23]. All results agree on finding protonated or deprotonated ions of the main analyte(s) as the predominant ion(s) (Fig. 1). Lower intensity ions can be seen, sometimes together with some fragments of the main analyte, formed by up-front collision induced dissociation, depending on the MS parameters. Interestingly other compounds, like excipients, are not sampled. An explanation could be that the polymers often used as excipients, like sugars or cellulose, are not desorbed and ionized under these conditions. Only the analyte(s) present in the tablet are desorbed, yielding clean full scan spectra.

It was observed that the signal intensity for the signal of ions of interest could change by a factor of around ten, depending on the positioning of the tablet under the spray and thus on the spot desorbed (Fig. 2). This change in signal can be explained by different surface roughness, with some kind of holes or peaks and microcrystals of analytes, yielding a non-constant desorption, but also by a relative inhomogeneity of the analyte on the surface as shown by IR imaging on pharmaceutical tablets [29]. It is not clear if there is another explanation for the observed phenomenon. No other results have reported this. One has to be conscious that not finding an expected compound is not a proof that it is absent, as it might be difficult or take more time to complete what could be considered an exhaustive desorption.

As tablets can be manufactured with a protective layer or film, we scrapped a 1- mm deep scratch on the commercial tablets before analysis. Simply cutting by hand divisible tablets is also possible, analyzing then their heart. Drugs sold as capsules cannot be directly analyzed by DESI-MS, the powder being hermetically sealed by the capsule. Nevertheless, the powder can simply be compressed, either alone or with an excipient such as cellulose, and then analyzed like standard tablets.

\section{DESI Mechanism}

Based on experimental conditions optimized for DESI, analytes can be divided into subgroups, for which it is believed that different mechanisms of ionization take place [28]. Some analytes are ionized by the charged droplets from the spray, a mechanism known as 'droplet pick-up'. The high velocity impact of the droplets (in the order of a few hundred meters per second in some cases) cause a small spread of the liquid on the surface, the analyte being extracted by the solvent into the droplet. This process is enhanced by increasing the velocity of the impacting droplets, i.e. increasing the nebulizing gas flow. These offspring droplets are then transferred to the mass spectrometer either by the gas of the spray, or by the vacuum aspiration at the mass spectrometer interface. Coulombic fission then generates gaseous ions by processes similar to those taking place in conventional ESI.

For other analytes, it is believed that their ionization does not use charged droplets. The main ion formation process could take place through charge or proton transfer from the solvent ions to the analyte molecule, either between gas phase ions and surface molecules, or between gas phase ions and gas phase molecules. The later could be enhanced when dealing with high vapor pres- sure analytes. The gas phase ions are produced from the volatile solvent by a standard corona-discharge ionization of the vapor. Desorption atmospheric-pressure chemical ionization (DAPCI) is then a suitable option for analyzing weakly polar compounds that do not ionize well by DESI.

Our experiments with pharmaceutical compounds favor the first mechanism. Qualitative and quantitative differences in ion intensities were clearly observed when using hydro-organic sprays containing either methanol or acetonitrile. For the majority of the twenty-one compounds investigated, the average counts per second (cps) value of the main ion was 5-20 times higher when a spray containing methanol was used, compared to acetonitrile [17]. Five compounds the same with both solvents and three gave better results with the spray containing acetonitrile (3-4 times more signal than with methanol). Direct, precise comparison between different DESI experiments remains difficult because of the varying geometry and surface states between the tablets, possibly affecting the MS signal. This general observation would have to be complemented with more compounds and other solvents, to see if the relationship between polarities of analytes and solvent plays the unique role. As the compounds investigated fall almost entirely in the electrospray group, with frequent nitrogen atoms that can be protonated, these differences can be explained by a changing content of analyte in the secondary charged droplet. The efficiency of what could be called the extraction of the analyte by the solvent changes depending on their respective polarities, and yields different ion intensities.

The original paper described a gas velocity in the order of a few hundred meters per second [3], however some papers de- 
scribe the use of conditions that seem to be more gentle, with modified commercial sources [23][24][26]. Although there is no direct comparison of gas velocities (arbitrary units of gas flow are given for commercial sources), it is clear that the conditions are not those of an almost supersonic spray. Further data on this point would be useful for a better general understanding of the relationship between gas velocity or inlet pressure and signal intensity. For some applications, a too high pressure spray could damage the surface, some examples being TLC plates or tablets with crumbly surfaces.

\section{Toxicological Screening}

It has been shown that DESI-MS can easily be used for the screening of drug tablets of various kinds, giving qualitative data on the main analyte(s). Drug content does not play a key role, since with tablets containing less than $1 \%(\mathrm{w} / \mathrm{w})$ of active ingredient we still observed the protonated ion [17]. This result was similar to other reports; contents of less than $1 \%(\mathrm{w} / \mathrm{w})$ [26] and even $0.1 \%(\mathrm{w} / \mathrm{w}$ ) [22] have been reported. Usual contents of pharmaceutical drug tablets are higher than $1 \%(\mathrm{w} / \mathrm{w})$. Nevertheless, there is the possibility of obtaining a false negative when low content tablets are desorbed on one spot only. The limit of sensitivity is also highly compound-dependent.

High-throughput experiments have been performed on pharmaceutical samples, with sampling rates as high as three tablets per second [22]. The set of tablets located on a moving belt was transported past the electrospray. A practical use with lower content tablets and as a method for quality assurance is questionable.

\section{Illicit Material Analysis}

As an extension to toxicological screening, illicit Ecstasy tablets have also been investigated [17][26]. Qualitative results by DESI-MS were in accordance with GC-MS and LC-MS results in the identification of the main analyte contained in the tablets. Active ingredients such as 3,4-methylene-dioxymethamphetamine (MDMA; Ecstasy), N-methyl-1-(1,3-benzodioxol-5yl)-2-butanamine (MBDB), amphetamine, methamphetamine, 4-methyl-thio-amphetamine (4-MTA) and only caffeine in fakes have been identified. These compounds have been common (with the exception of 4-MTA) for a while in Western Switzerland in illicit tablets sold as 'Ecstasy' [30]. As content of active ingredient in illicit drug tablets is in the same range as pharmaceutical tablets, we see no reason why almost any tablet could not be analyzed by DESI-

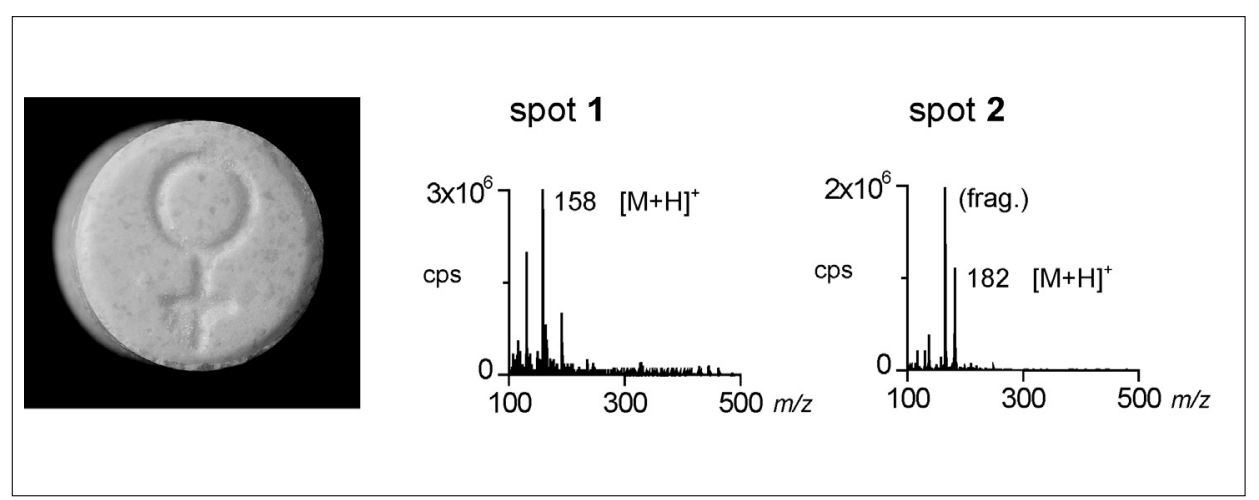

Fig. 3. DESI-MS analysis of a seized Ecstasy tablet, with different ions sampled from different locations

MS. If some minor analytes must be identified, GC-MS and LC-MS are still methods of choice, as some of these analytes are missed when sampled by DESI-MS.

Cannabis plant material has also been subjected to DESI-MS, with the in situ sampling of cannabinoids directly from dried pieces of marijuana leaves [26]. Two other examples, with the detection of alkaloids in plant tissue under these ambient conditions, show the feasibility of the approach, despite the very few data published up to now [3][25].

\section{Chemical Imaging}

As the sample can be freely moved under the spray during mass spectra acquisition and since the desorbed spot is confined, chemical imaging can be done by DESI-MS. A simple example in the analysis of tablets was done on Ecstasy tablets (Fig. 3). This was chosen because this kind of tablet usually shows great inhomogeneities, as they are generally homemade. Depending on the position of the tablet under the spray, different analytes could be sampled from different spots. Correlating visual inspection of inhomogeneities with different mass spectra could illustrate the formal advantage that surface analysis techniques exhibit over solution-based techniques, as this kind of information is lost in the latter case. Mass spectrometric profiling of intact mouse-pancreas, ratbrain or metastatic human-liver adenocarcinoma tissues has been demonstrated, showing the potential of in vivo/in situ applications of DESI-MS [21].

\section{Perspectives}

DESI-MS has rapidly gained in interest in the first years since its introduction. The use of DESI-MS for the rapid toxicological screening of a large variety of commercial drugs, illicit Ecstasy tablets, and compressed powders was demonstrated, correlated with similar results obtained by other groups. Sampling under ambient conditions without any sample preparation or chromatographic step, instant response, imaging capability, and the fact that DESI experiments can be performed in parallel with other experiments are great features of this technique, although some points still need to be assessed. Among these we could mention the source metrics and the influence of the spraying solvent, problems related to matrix suppression, the quantitative aspect, and the feasibility of using this setup in routine applications. Further work is ongoing in our group to assess its advantages and limitations in other pharmaceutical analysis applications. DESI-MS, along with some of the closely related desorption ionization techniques, shows the great potential of these ambient desorption techniques as an alternative to solution-based analysis and, in some cases, to using chromatographic equipment coupled to mass spectrometers.

Received: February 19, 2006

[1] V.V. Laiko, M.A. Baldwin, A.L. Burlingame, Anal. Chem. 2000, 72, 652.

[2] V.V. Laiko, S.C. Moyer, R.J. Cotter, Anal. Chem. 2000, 72, 5239.

[3] Z. Takats, J.M. Wiseman, B. Gologan, R.G. Cooks, Science 2004, 306, 471.

[4] J. Wei, J.M. Buriak, G. Siuzdak, Nature 1999, 399, 243.

[5] R.B. Cody, J.A. Laramee, H.D. Durst, Anal. Chem. 2005, 77, 2297.

[6] C.N. McEwen, R.G. McKay, B.S. Larsen, Anal. Chem. 2005, 77, 7826.

[7] J. Shiea, M.Z. Huang, H.J. Hsu, C.Y. Lee, C.H. Yuan, I. Beech, J. Sunner, Rapid Commun. Mass Spectrom. 2005, 19, 3701.

[8] M. Theune, W. Esser, K.F. Druschky, E. Interschick, H. Patscheke, Nervenarzt 1999, 70, 1094.

[9] M. Ohtsuji, J.S. Lai, S.R. Binder, T. Kondo, T. Takayasu, T. Ohshima, J. Forensic Sci. 1996, 41, 881.

[10] Y. Roggo, A. Edmond, P. Chalus, M. Ulmschneider, Anal. Chim. Acta 2005, 535, 79. 
[11] R.C. Schneider, K.A. Kovar, Forensic Sci. Int. 2003, 134, 187.

[12] N. Sondermann, K.A. Kovar, Forensic Sci. Int. 1999, 106, 147.

[13] S.E.J. Bell, J.R. Beattie, J.J. McGarvey, K.L. Peters, N.M.S. Sirimuthu, S.J. Speers, J. Raman Spectrosc. 2004, 35, 409.

[14] A.B. Moreira, H.P.M. Oliveira, T.D.Z. Atvars, I.L.T. Dias, G.O. Neto, E.A.G. Zagatto, L.T. Kubota, Anal. Chim. Acta 2005, 539, 257.

[15] A.B. Moreira, I.L.T. Dias, G. Oliveira Neto, E.A.G. Zagatto, L.T. Kubota, Anal. Chim. Acta 2004, 523, 49.

[16] E. Balchin, D.J. Malcolme-Lawes, I.J. Poplett, M.D. Rowe, J.A. Smith, G.E. Pearce, S.A. Wren, Anal. Chem. 2005, 77, 3925.

[17] L.A. Leuthold, J.F. Mandscheff, M. Fathi, C. Giroud, M. Augsburger, E. Varesio, G. Hopfgartner, Rapid Commun. Mass Spectrom. 2006, 20, 103.

[18] G.J. Van Berkel, M.J. Ford, M.A. Deibel, Anal. Chem. 2005, 77, 1207.

[19] Z. Takats, I. Cotte-Rodriguez, N. Talaty, H. Chen, R.G. Cooks, Chem. Commun. 2005, 1950.

[20] I. Cotte-Rodriguez, Z. Takats, N. Talaty, H. Chen, R.G. Cooks, Anal. Chem. 2005, 77, 6755 .
[21] J.M. Wiseman, S.M. Puolitaival, Z. Takats, R.G. Cooks, R.M. Caprioli, Angew. Chem. Int. Ed. 2005, 44, 7094.

[22] H. Chen, N.N. Talaty, Z. Takats, R.G. Cooks, Anal. Chem. 2005, 77, 6915.

[23] D.J. Weston, R. Bateman, I.D. Wilson, T.R. Wood, C.S. Creaser, Anal. Chem. 2005, 77, 7572 .

[24] J.P. Williams, J.H. Scrivens, Rapid Commun. Mass Spectrom. 2005, 19, 3643.

[25] N. Talaty, Z. Takats, R.G. Cooks, Analyst 2005, 130, 1624.

[26] S.E. Rodriguez-Cruz, Rapid Commun. Mass Spectrom. 2006, 20, 53.

[27] T.J. Kauppila, J.M. Wiseman, R.A. Ketola, T. Kotiaho, R.G. Cooks, R. Kostiainen, Rapid Commun. Mass Spectrom. 2006, 20 , 387.

[28] Z. Takats, J.M. Wiseman, R.G. Cooks, J. Mass Spectrom. 2005, 40, 1261.

[29] P. Seitavuopio, J. Rantanen, J. Yliruusi, Eur. J. Pharm. Biopharm. 2005, 59, 351.

[30] C. Giroud, M. Augsburger, F. Sadeghipour, E. Varesio, J.-L. Veuthey, L. Rivier, Praxis 1997, 86, 510. 